\title{
Shadow Map Construction using 2D Homography
}

\author{
Trinh Hien Anh, Trinh Xuan Hung
}

Abstract: In computer graphic applications, shadow plays an important role in expressing the reality of an object. Shadow shows the relationship between objects in space. Shadow map is one of the methods that meet the demand simplification in implementation and speed. This method made an alias around the border of shading. In this paper, we proposed a method using a $2 D$ homography transformation. This method reduces the unused area in the shadow maps, so it can help to minimize the alias. With the experiment in VanMieu Tran Bien-a Vietnamese historical place, we compare the method with others.

Keywords: shadow map, augmented reality, GPU.

\section{INTRODUCTION}

Shadow plays an important role in a computer graphics application. Without shadow, we meet difficulty in detecting the spatial relationship of objects in the space. Researches on creating the shadow for objects in the 3D environment are divided into 2 mains approach: Shadow Map (SM)[1] and Shadow Volume (SV)[7]. Shadow Volume based on geometry, it requires all information of the polygon meshes of objects in the scene to compute the silhouette. This approach takes time and difficult to set up. Meanwhile, the other approach, shadow mapping is easier to set up and faster. But this cause the alias in the shadow areas, see figure 1.

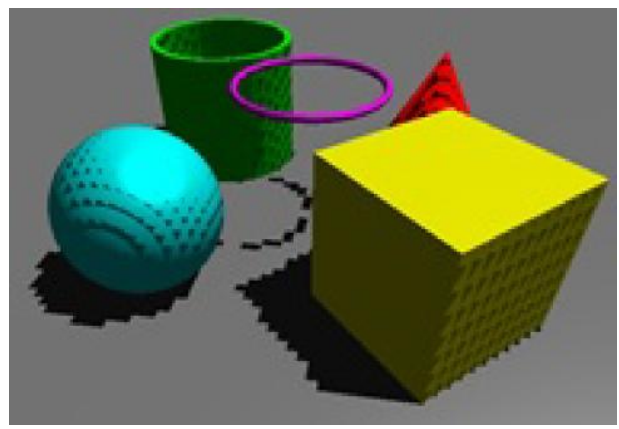

Fig. 1. Alias of the shadow

At the boundary of the shadow area, the alias appear due to the incompatibility between the camera viewing and the storage of shadow maps. With the aim at overcoming this, researchers study to optimize the shadow map, such as: ASM- Adaptive Shadow Map [2], Perspective Shadow Map-PSM [8], and Light Space Perspective Shadow MapsLSPSM [10]. ASM was first introduced by R.Fernado et al [2] they remove aliasing by using hierarchical shadow maps. These maps built based on image resolution. Then, they used dynamically with each pixel in the camera view.

Revised Manuscript Received on April 30, 2020.

* Correspondence Author

Trinh Hien Anh, Virtual Reality Department, Institute of Information Technology, Hanoi, Vietnam. Email: hienanh@ioit.ac.vn

Trinh Xuan Hung*, Virtual Reality Department, Institute of Information Technology, Hanoi, Vietnam. Email: trxhung@ioit.ac.vn

Thanks for the project B2018-TNA-61, VAST’0119

(C) The Authors. Published by Blue Eyes Intelligence Engineering and Sciences Publication (BEIESP). This is an open access article under the CC BY-NC-ND license (http://creativecommons.org/licenses/by-nc-nd/4.0/)
This technique requires a number of shadow maps with different resolutions so it is not suitable with a real time and complex scene. Works in $[5,6,9,11]$ are like Fernado's approach, they use different criteria to divide the components of the shadow maps.

PSM- a technique that uses Post-perspective projection proposed by Stamminger et all to increase the size of the object near the project plane. This technique could remove the alias and only accuracy when the light and the view are perpendiculars.

Wimmer and his colleagues used a secondary projective from the camera, this perpendicular with the light direction. This technique call LSPSM, helps to overcome the disadvantage of PSM. In fact, it is only suitable for the parallel light source.

Our proposed method also solves the problem of optimizing the shadow map based on a 2D Homographic projection. This transforms the observation space into the standard square space on the shadow map. The paper organizes as follows. Section 2 we present our proposed method to enhance the shadow's quality using Homography 2D. In Section 3 we do the Experiment and assess the proposed method. Conclusion of the paper is in Section 4.

\section{THE PROPOSED METHOD}

We propose a method using 2D Homograph projection to optimize the shadow map. With this $2 \mathrm{D}$ projection, the observation space mapped to a standard square in the shadow map. As we can see in figure 2, the dark line depicts the projection of the camera view. The blurred trapezoid covers the projection area. The projection area occupies a small part in the shadow map, that is the reason causes the alias at the boundary of the shadow region. Researches on reduce the alias are concentrate on minimizing the unused area (the area between the blurred line and the square).

The proposed process to create a shadow for the scene includes these below main steps:

Step 1: Draw the scene with the camera parameters, that include: position, rotation angle and the projection parameters.

Step 2: Draw the shadow map with the parameter of the camera and the light source.

Step 3: Draw the shadow with the shadow map in Step 2 and depth map in Step 1.

In step 2 and 3 we use 2D homography to solve the problems.

Published By:

Blue Eyes Intelligence Engineering

\& Sciences Publication

(C) Copyright: All rights reserved.

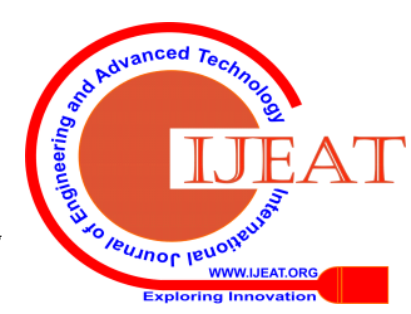




\section{Shadow Map Construction using 2D Homography}

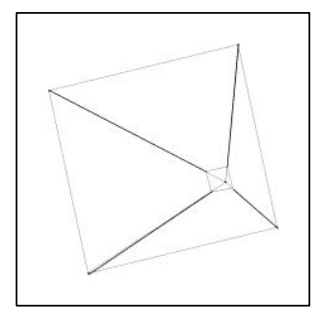

(a)

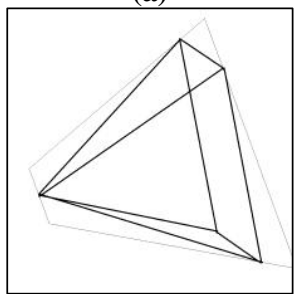

(c)

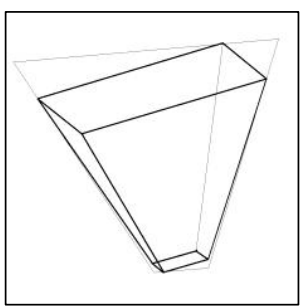

(b)

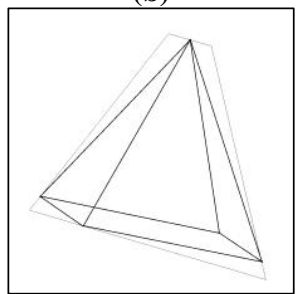

(d)
Fig. 2. Camera views project into the light source views

\section{A. Building a 2D Homography transform}

As mentioned, we aim at building a transformation that minimizes the unused space and enhancing the space of object near the camera view. The space of object near camera is a convex in $2 \mathrm{D}$ plane. And we know that a $2 \mathrm{D}$ homography transform a convex to another convex. So, in the next step, we find out a projected matrix to meet the demand.

Frist, we find out the smallest trapezoid that has a smaller base near the projection plane than the large one. This trapezoid covers the camera space in the light projection. After that, we use the Homography projection to change this trapezoid into the basic square

$$
\mathrm{P}^{0}=\{(-1,-1),(1,-1),(1,1),(-1,1)\} \text {. }
$$

The Homography projection is the $3 \times 3$ matrix that can be found out after solve the systems of linear equation (1) below:

$\left\{\sum_{i=0}^{2} \quad P_{0, i} M_{0, i}+M_{0,2}-P_{0,0}^{0}\left(\sum_{i=0}^{2} \quad P_{0, i} M_{2, i}\right)-M_{2,2} P_{0,0}^{0}=\right.$ $0 \sum_{i=0}^{2} \quad P_{0, i} M_{1, i}+M_{1,2}-P_{0,1}^{0}\left(\sum_{i=0}^{2} \quad P_{0, i} M_{2, i}\right)-M_{2,2} P_{0,1}^{0}=$

$0 \sum_{i=0}^{2} \quad P_{1, i} M_{0, i}+M_{0,2}-P_{1,0}^{0}\left(\sum_{i=0}^{2} \quad P_{1, i} M_{2, i}\right)-M_{2,2} P_{1,0}^{0}=0 \quad \vdots$

$\sum_{i=0}^{2} \quad P_{3, i} M_{1, i}+M_{1,2}-P_{3,1}^{0}\left(\sum_{i=0}^{2} \quad P_{3, i} M_{2, i}\right)-M_{2,2} P_{3,1}^{0}=0 M_{2,2}=$ $1 \quad(1)$

In which, $\mathrm{P}^{0}$ is the basic square; $\mathrm{P}$ is the smallest trapezoid that cover the camera space. $\mathrm{M}$ is the matrix that we find.

\section{B. Create the shadow map}

The process of creating a shadow map is shown in figure 3. There are 4 main steps in the process.

Step 1: Estimate the matrix that transforms object from the camera view into a light source. This call Camera to Light Matrix- C2LM.

Step 2: Estimate the projection matrix to build the shadow map from the light and camera. In this step we use the Light Projection Matrix-LPM.

Step 3: Estimate the 2D Homography projection base on the camera and the light view with camera projection matrix (CPM), LPM and C2LM.

Step 4: Render the scene in GPU with pipeline mode as shown in figure 3 .

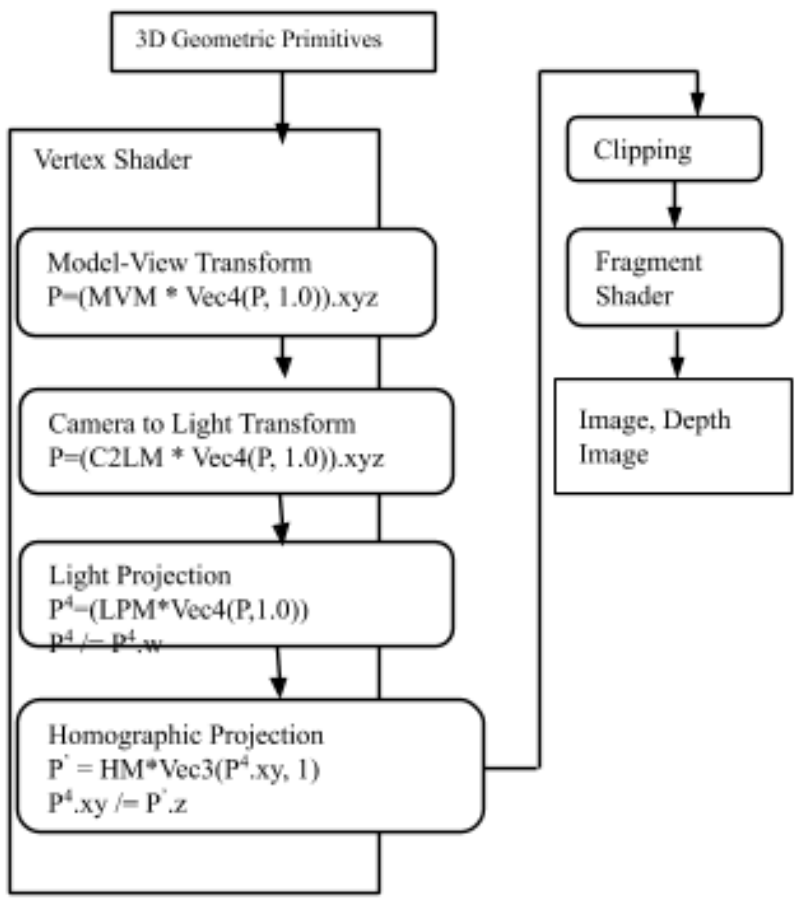

Fig. 3. Diagram of drawing shadow map

The input for the diagram in figure 3 is the set of polygons that used to create models. With each model we have an appropriate transformation matrix into camera view. After the process, we have a depth image. This store in a 2D texture.

\section{Draw the object's shadow}

An object's shadow could be drawn by two ways that mention below. One, draw each object in the scene. These objects are drawn with the shadow map and matrices. Then, they combine with the scene from the camera to have the final image. Second, using the depth image. With each point in the depth image, we calculate the appropriate point in the camera view. This point is then transformed into the light view to compare with the shadow map to decide whether it belongs to the shadow region or not.

In this paper, we use the second one. We do this process in GPU with Render Pipeline.

Input to the process is a basic square; estimate matrices; depth map (depM) and shadow map (sdM). The output of the process is the scene with the object's shadow. The main steps are in Fragment Shader, below:

Frist, we do the Camera Projection Invert with the equation:

$$
\begin{gathered}
P . z=\text { texture }(\operatorname{dep} M, P) \cdot x y P^{4}=\operatorname{ICPM} \times \operatorname{Vec} 4(P, 1) P \\
=P^{4} \cdot x y z / P^{4} \cdot w
\end{gathered}
$$

After this step, all points in the scene will be transferred into the camera view. These points then continue projecting to the light view by the equation below

$$
\begin{gathered}
P=(C 2 L M \times \operatorname{Vec} 4(P, 1.0)) \cdot x y z \\
\text { And do light Projection } \\
P^{4}=L P M \times \operatorname{Vec} 4(P, 1.0) P^{4} /=P^{4} . w
\end{gathered}
$$

Homographic Projection 


$$
\begin{gathered}
P^{\prime}=H M \times \operatorname{Vec} 3\left(P^{4} \cdot x y, 1\right) \\
P^{4} \cdot x y /=P^{\prime} . z
\end{gathered}
$$

Finally, we compare the depth to deceide wether the point belongs to the shadow or not

$$
s d=\operatorname{shadow}\left(\operatorname{sd} M, P^{4} \cdot x y z\right)
$$

\section{Analytics}

Some strong points of our proposed method.

- Easy to implement. Instead of calculating all points and bounding box in 3D space $[8,9,10]$, we calculate them in $2 \mathrm{D}$ space, the projection plane of the light source.

- The complexity of the computation has been reduced due to the use of the smallest trapezoid cover the camera view.

- Proposed method can be used for the point light directly while ensuring the quality of the shadow.

We use GPU to calculate all 2D matrices using in the process so the time is acceptable. Our proposed method needs a computer that supports Vertex Shader and Fragment Shader, that is the weak point.

\section{EXPERIMENTS}

We use the scene of Van Mieu Tran Bien, one of Vietnamese historical places to do the experiment. This scene includes more than 1.2 billion polygons. The proposed method is written in $\mathrm{C}++$ and OpentGL and installed in Core I7 4790 with NVIDIA GeForceFX 630.

The data were tested with the original shadow map, the LSPSM and proposed method with the resolution of $256 \times 256 ; \quad 512 \times 512 ; \quad 1024 \times 1024$ and $2048 \times 2048$ sequentially.

In figure 4, we show the shadow map in the comparison between the original and the HSM. In which, (a) show the difference between the observation direction and light source direction is large enough to build a trapezoid that covers the observation view in light source space. And (c), the light source is nearly partial with the camera. In both cases, the proposed method shows the advantage by comparing between $(\mathrm{a}, \mathrm{c})$ and $(\mathrm{b}, \mathrm{d})$.

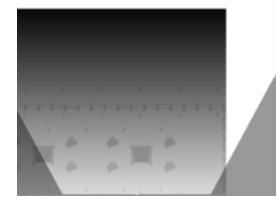

(a)

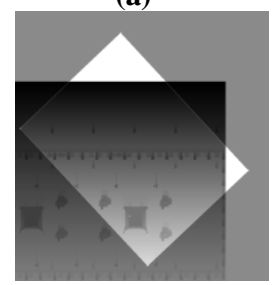

(c)

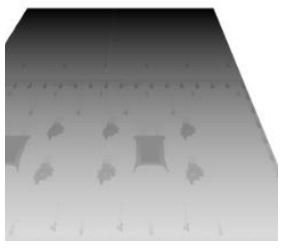

(b)

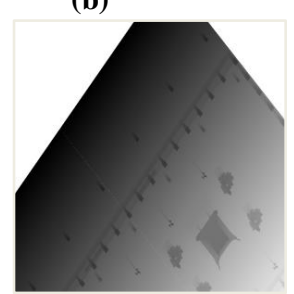

(d)

Fig. 4.The shadow map of the original and HSM. (a) and (c) is the shadow map from the camera view, (b) and (d) is the shadow map after applying the HSM.

In figure 5, we show the shadow of different algorithms with the resolution of the shadow map $512 \times 512$. In which,

(a) is for the traditional algorithm (b) and (c) is the result of LSPSM and HSM. We easily find out that the quality of the shadow is nearly the same. HSM is a little bit better.

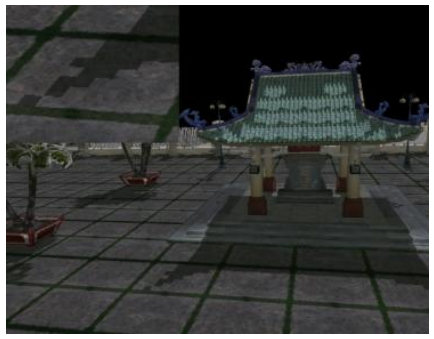

(a)

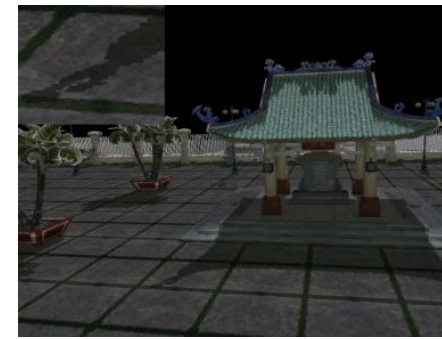

(b)

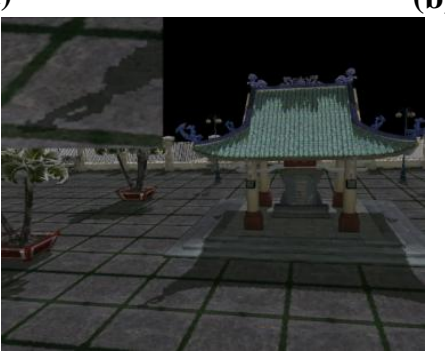

(c)

Fig. 5. Shadow with resolution of shadow map 512x512 (a) Traditional method (b) LSPSM (c) HSM

We assess the speed rate of the algorithm by calculating number of frames render randomly in 10 minutes in Van Mieu Tran Bien. In figure 5 we compare the speed rate of different algorithms. With this diagram, we see that the speed rate of proposal method, HSM is similar to the traditional algorithm ans LSPSM

In figure 6, we compare the performance of the original shadow map with LSPSM and the proposed method. We do the assessment in 10 minutes with the camera translating randomly in a certain range of Van Mieu Tran Bien. The result shows that the frame rate of the proposed method similar to traditional and LSPSM ones.

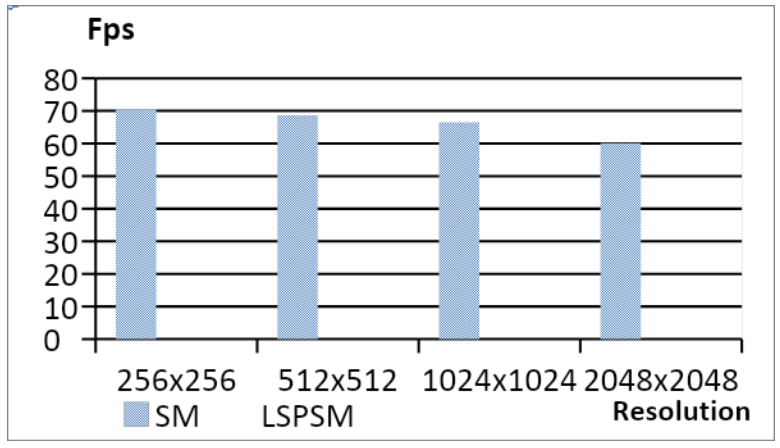

Fig. 6.Compare the frame rate between the algorithms

\section{CONCLUSION}

In the paper, we have proposed a technique to enhance the shading effect with a new shadow map by applying a twodimensional Homography transformation on the shadow map image to optimize the storage space on the map. The HSM aims at minimizing the redundant areas and increase the size of areas near the projection plane. It shows the ability to reduce alias in the shadow region. 


\section{Shadow Map Construction using 2D Homography}

In comparison with the traditional algorithm and LSPSM, with the scene of Van Mieu Tran Bien, the proposed method confirms the advantage in reducing the alias in an acceptable time.

\section{REFERENCES}

1. Everitt, Cass; Rege, Ashu; Cebenoyan, Cem. "Hardware shadow mapping". White paper, nVIDIA, 2001, 2.

2. Fernado, Randima, et al. "Adaptive shadow maps". In: Proceedings of the 28th annual conference on Computer graphics and interactive techniques. ACM, 2001. p. 387-390.

3. Gumbau, Jesús, et al. "Shadow map filtering with gaussian shadow maps". In: Proceedings of the 10th International Conference on Virtual Reality Continuum and Its Applications in Industry. ACM, 2011. p. 7582.

4. Hoshang Kolivand, Mohd Shahrizal Sunar, Ayman Altameem, Amjad Rehman and Mueen Uddin "Shadow Mapping Algorithms: Applications andLimitations, Appl. Math. Inf. Sci. 9, No. 3, 1307-1315 (2015)

5. Lefohn, Aaron E.; Sengupta, Shubhabrata; Owens, John D. Resolutionmatched shadow maps. ACM Transactions on Graphics (TOG), 2007, 26.4: 20.

6. Lloyd, D. Brandon, et al. "Logarithmic perspective shadow maps". ACM Transactions on Graphics (TOG), 2008, 27.4: 106.

7. e. In: ACM Transactions on Graphics (TOG). ACM, 2003. p. 521-526.

8. Stamminger, Marc; Drettakis, George. "Perspective shadow maps". In: ACM transactions on graphics (TOG). ACM, 2002. p. 557-562.

9. Trịnh Xuân Hùng, Trịnh Hiền Anh, Đỗ Văn Thiện, Hà Mạnh Toàn "Một kỹ thuật tăng tốc tính toán bóng cho hiển thị các đối tượng 3D trong Thực tại ảo" Kỷ yếu hội thảo quốc gia về "Nghiên cứu cơ bản và ứng dụng công nghệ thông tin FAIR“" Thái Nguyên 2014, tr 318-321

10. Wimmer, Michael, Scherzer, Daniel; Purathofer, Werner. "Light space perspective shadow maps. Rendering Techniques", 2004, 2004: 15th.

\section{AUTHORS PROFILE}

Trinh Hien Anh did her B.Tech in Computer and Math in 2000 from Thang Long University, Hanoi. She did her M.Tech in in Software Engineering in 2011. She has had 19 years experience and currently working in Information Of Information TechnologyVAST. Her research interests are in the area of Fuzzy Logic, Computer Network, Image Processing, and Virtual Reality

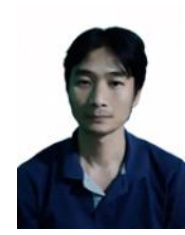

Trinh Xuan Hung did his B.Tech in Computer Science and Engineering in 2004 from College of Information Technology ,Hanoi. He did his M.Tech in Software Engineering in 2011. He has had 12 years experience and currently working in Information Of Information Technology-VAST. His research interests are in the area of Computer Network, Image Processing, and Virtual Reality

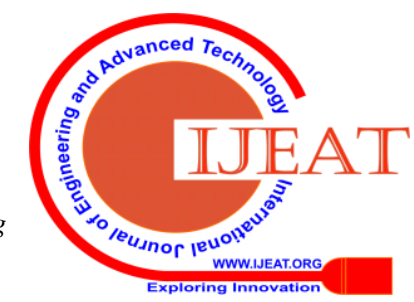

